\title{
Correlation of Serum Opsonic Activity in Cystic Fibrosis with Colonization and Disease State: Measurement of Opsonins to Pseudomonas aeruginosa by Neutrophil Superoxide Anion Generation
}

\author{
JAMES G. BENDER, ALFRED L. FLORMAN AND DENNIS E. VAN EPPS \\ Departments of Pathology [J.G.B., D.E.V.E.], Medicine [D.E.V.E.], and Pediatrics [A.L.F.], University of New \\ Mexico School of Medicine, Albuquerque, New Mexico 87131
}

\begin{abstract}
Serum from patients with cystic fibrosis and normal controls was used to opsonize mucoid and nonmucoid Pseudomonas aeruginosa particles. Opsonic activity was then determined by measuring the production of superoxide anion $\left(\mathrm{O}_{2}^{-}\right)$from normal neutrophils stimulated with the opsonized particles. Without any opsonization, mucoid $P$. aeruginosa stimulated significantly more $\mathrm{O}_{2}^{-}$ than nonmucoid $P$. aeruginosa. Responses to nonmucoid $P$. aeruginosa observed with heat-inactivated serum from patients with cystic fibrosis were significantly higher $(p=$ 0.008 ) than those observed with heat-inactivated control sera. Comparisons made between patients who were colonized with $P$. aeruginosa and those who were not showed that heat activated serum from colonized patients had significantly higher levels of opsonic activity than heat inactivated serum from patients who were not colonized. These differences were observed with either mucoid or nonmucoid $P$. aeruginosa. A negative correlation was also observed between opsonic activity and clinical status measured by Schwachman scores of colonized patients. These data indicate that in patients colonized with $P$. aeruginosa the deterioration of their clinical status correlated with increased opsonic activity reflected in the oxidative burst response of neutrophils. (Pediatr Res 22: 383-388, 1987)
\end{abstract}

Abbreviations

CF, cystic fibrosis

HBSS, Hanks' balanced salt solution

PBS, phosphate-buffered saline

PMN, polymorphonuclear cells

$\mathrm{CF}$ is characterized by progressive inflammatory lung disease involving the accumulation of neutrophils. Patients with $\mathrm{CF}$ have relatively normal pulmonary function until the onset of Pseudomonas aeruginosa colonization of the respiratory tract (1). From this point, exacerbations of obstructive pulmonary disease ultimately result in pulmonary failure. One model for the progression of this lung disease involves a hyperimmune

Received October 14, 1986; accepted May 5, 1987

Address for correspondence James G. Bender, Ph.D., Department of Pathology BRF 323, University of New Mexico School of Medicine, Albuquerque, NM 87131.

Supported in part by DHHS Grants MCJ-000949-11 from the Maternal Child Health Section and CA 20819 from the National Cancer Institute. J. G. B. was the recipient of a postdoctoral fellowship from the Cystic Fibrosis Foundation. humoral response to $P$. aeruginosa resulting in immune complex formation and the activation of neutrophils $(2,3)$. Activated neutrophils in turn infiltrate the lung, interact with the microbes present, and contribute to its damage through the secretion of oxygen radicals and degradative enzymes (4). The major evidence which supports this model has been the observation that high antibody titers to $P$. aeruginosa are correlated with a poorer clinical state $(5,6)$. The role of these antibodies in stimulatory oxygen radical production in neutrophils is not well defined. Recent studies by Fick et al. (7) have indicated that IgG antibodies produced to $P$. aeruginos $a$ are defective in their ability to support phagocytosis by alveolar macrophages. However, when neutrophils have been examined no defect in phagocytosis has been observed. Since pulmonary clearance of $P$. aeruginosa is mainly due to neutrophils rather than alveolar macrophages (8), the interaction of neutrophils with $P$. aeruginosa and the subsequent production of oxygen radicals is likely to be important in the progression of this lung disease.

The activation of neutrophils by microbial particles is enhanced by opsonic factors present in the circulation. These factors include IgG and complement components such as C3b. The measurement of oxygen radical production has been widely used to quantify opsonic activity $(9,10)$. We therefore measured the generation of superoxide anion from normal neutrophils stimulated by heat-killed mucoid and nonmucoid $P$. aeruginosa organisms. These particles had been opsonized with serum from $\mathrm{CF}$ patients and normal controls. When heat inactivated to denature complement components, sera from some CF patients stimulated more superoxide anion release. CF patients colonized with $P$. aeruginosa gave a higher response than patients who were not colonized, and there was an inverse correlation between the opsonic activity and clinical status.

\section{MATERIALS AND METHODS}

Preparation of neutrophils. Human peripheral blood neutrophils were separated from the heparinized venous blood of normal adult volunteers using Ficoll-hypaque gradient centrifugation to remove mononuclear leukocytes (11). The cell pellet containing erythrocytes and neutrophils was resuspended in HBSS (GIBCO, Grand Island, NY) and mixed with Plasmagel (Cellular Products, Buffalo, NY) at a concentration of $1 \mathrm{ml}$ Plasmagel per $3 \mathrm{ml}$ of blood. The leukocyte-rich supernate obtained after $30 \mathrm{~min}$ of $37^{\circ} \mathrm{C}$ incubation was then centrifuged and the cell pellet mixed with $4^{\circ} \mathrm{C}$ distilled water for $15 \mathrm{~s}$ to lyse residual erythrocytes. Cells were then washed twice and resuspended in $0.01 \mathrm{M}$ PBS with $1 \mathrm{mM} \mathrm{Ca}^{++}$and $1 \mathrm{mM} \mathrm{Mg}^{++}, \mathrm{pH}$ 
7.4. Differential counts on Wright's stained cells indicated greater than $95 \%$ neutrophils.

Serum samples. Serum was obtained from 16 CF patients (see patient summary, Table 1) at the time of their visit to the CF Clinic at the University of New Mexico Hospital. All of the patients were ambulatory at the time of the blood collection. Serum was also collected from normal healthy controls. All samples were stored at $-70^{\circ} \mathrm{C}$ until used.

Preparation of bacteria. A nonmucoid strain of $P$. aeruginosa (ATCC27853) and four mucoid stains isolated from sputum cultures from $\mathrm{CF}$ patients were obtained from the microbiology laboratory at the University of New Mexico Hospital. Pure cultures of the organisms were streaked onto agar plates containing $2 \%$ sheep blood and harvested after a 24 -h incubation at $37^{\circ}$

Table 1. Patient data

\begin{tabular}{cccc}
\hline Patient & Sex & Age & $\begin{array}{c}\text { Schwachman } \\
\text { Score }\end{array}$ \\
\hline Patients colonized & with & Pseudomonas \\
I & M & 12.9 & 52 \\
2 & M & 12.3 & 52 \\
3 & M & 10.6 & 58 \\
4 & M & 14.7 & 62 \\
5 & M & 8.9 & 72 \\
6 & M & 5.6 & 83 \\
7 & F & 16.2 & 87 \\
8 & M & 10.1 & 90 \\
Means & & 11.5 & 69.5 \\
& & & \\
Patients without Pseudomonas colonization & \\
9 & M & 6.5 & 60 \\
10 & F & 13 & 75 \\
11 & M & 6.4 & 77 \\
12 & M & 24.5 & 77 \\
13 & M & 4.3 & 78 \\
14 & M & 9.5 & 82 \\
15 & M & 9.2 & 85 \\
16 & F & 10.1 & 85 \\
Means & & 11 & 79.9 \\
\hline
\end{tabular}

C. It was determined at this point that viable organisms would reduce cytochrome $\mathrm{c}$ thereby interfering with the measurement of superoxide anion production. To prevent this interference the organisms were heat killed by boiling for $1 \mathrm{~h}$ and washed twice in PBS. The particles were then resuspended to give an OD of 0.6 at $620 \mathrm{~nm}\left(\sim 10^{8}\right.$ particles $\left./ \mathrm{ml}\right)$. These preparations were stored at $-70^{\circ} \mathrm{C}$ and thawed just prior to use.

Opsonization protocol. The $P$. aeruginosa particle suspension, $0.4 \mathrm{ml}$, prepared above was placed into $1.5 \mathrm{ml}$ Eppendorf microcentrifuge tubes, centrifuged at $10,000 \mathrm{rpm}$ for $20-30 \mathrm{~s}$, and the supernate removed with a Pasteur pipette. The diluted sample of serum was added and the particles were resuspended by vortexing. The samples were rotated at $37^{\circ} \mathrm{C}$ for $30 \mathrm{~min}$, washed twice in PBS, and resuspended to $0.4 \mathrm{ml}$. These preparations were then used to stimulate neutrophil superoxide anion generation.

Superoxide anion assays. Generation of superoxide by neutrophils was determined using a modification of the method of Pick and Mizel (12) measuring superoxide dismutase inhibitable cytochrome $\mathrm{c}$ reduction in 96 well tissue culture plates (Falcon, Oxnard, CA). This assay utilizes $5 \times 10^{5}$ neutrophils/well in 80 $\mu \mathrm{M}$ cytochrome $\mathrm{c}$ with a final volume in each well of $0.2 \mathrm{ml}$. Stimulation of neutrophils was initiated at $37^{\circ} \mathrm{C}$ by the addition of $50 \mu \mathrm{l}$ of serum-treated bacterial particles and the absorbance at $550 \mathrm{~nm}$ was monitored using a Dynatech automated ELISA reader (MR580) (Santa Monica, CA) and compared to identical wells containing $50 \mu \mathrm{g} / \mathrm{ml}$ superoxide dismutase (Sigma, St. Louis, MO). The maximal absorbance change was determined to occur after 20-30 min of incubation and was used to calculate the amount of superoxide anion produced using the molar extinction coefficient of $21 \times 10^{3} \mathrm{M}^{-1} \mathrm{~cm}^{-1}$ (13).

Clinical evaluation of patient status. In order to determine the severity of disease in our cystic fibrosis patients, the system of clinical scoring devised by Schwachman and Kulczycki (14) was used. The evaluation is based on four items: 1) general activity, 2) physical findings, 3) nutritional status, and 4) findings on chest $x$-ray. Each item was given 25 points and a total of 100 points was a perfect score. The status of a patient was considered excellent when the score was over 85 , good when the score was between 71 and 85 , mild when between 56 and 70 , moderate between 41 and 55 , and severe when 40 or less. The scores were assigned by one of us (ALF) before the results of the neutrophil

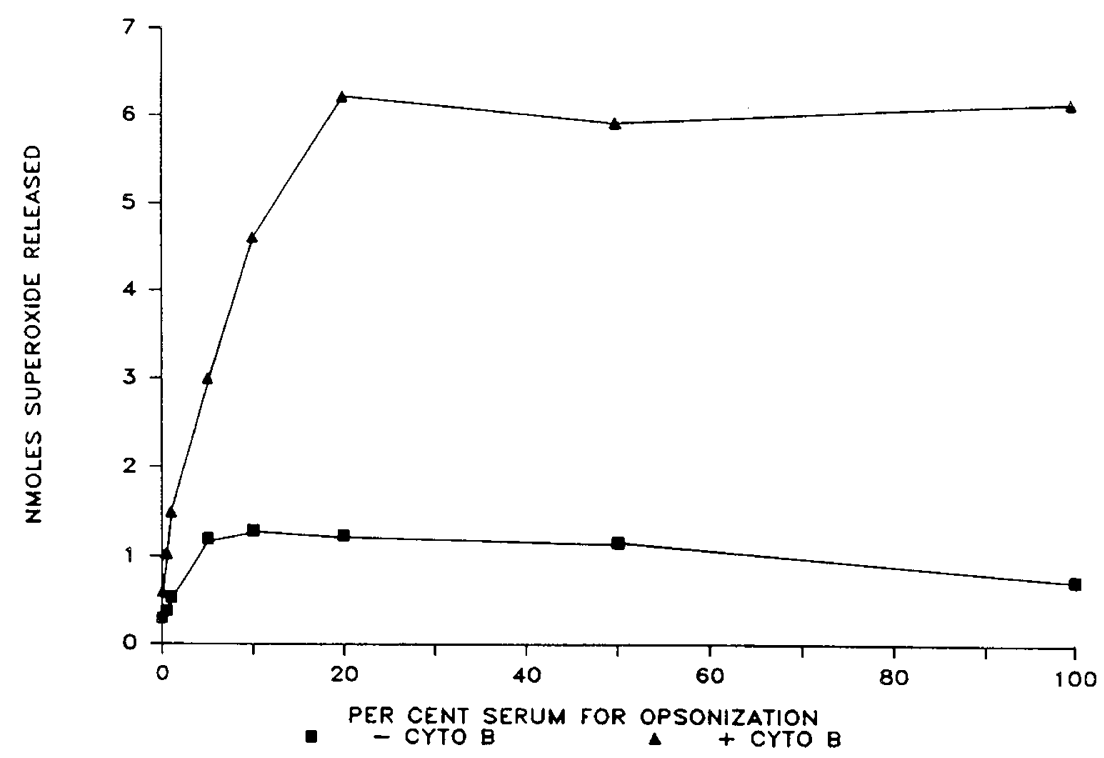

Fig. 1. Effect of control serum concentration and cytochalasin B treatment on the release of superoxide anion from neutrophils stimulated with nonmucoid $P$. aeruginosa. Heat-inactivated $P$. aeruginosa organisms were opsonized with varying concentrations of normal serum for 30 min at $37^{\circ} \mathrm{C}$. The particles were washed by centrifugation and used to stimulate superoxide anion release from normal neutrophils which had been pretreated with $(\mathbf{\Delta})$ or without $(\square) 5 \mu \mathrm{g} / \mathrm{ml}$ cytochalasin B for $5 \mathrm{~min}$ at $37^{\circ} \mathrm{C}$. 
superoxide anion generation studies were known to him. They reflect the patient's status on the day serum was obtained.

\section{RESULTS}

Titration of serum opsonic activity and the effects of cytochalasin $B$ on neutrophil superoxide production. Initial experiments were performed to determine the serum concentration which would be optimal for the opsonization of $P$. aeruginosa. In addition, neutrophils were pretreated with and without cytochalasin B $(5 \mu \mathrm{g} / \mathrm{ml})$ for $5 \mathrm{~min}$ at $37^{\circ} \mathrm{C}$. Cytochalasin B disrupts microfilaments, thereby preventing internalization of superoxide (15) and phagocytosis (16). This was included to enhance the extracellular production of superoxide as well as to minimize effects from differences in the phagocytosis of the organisms. As shown in Figure 1, increasing the concentration of serum used to opsonize the control nonmucoid $P$. aeruginosa resulted in enhanced production of superoxide anion. This enhancement reached a maximum at $20 \%$ serum. Treatment of neutrophils with cytochalasin B also greatly enhanced the superoxide anion response. Similar results were obtained when mucoid $P$. aerugi- nosa particles were used (data not shown). On the basis of these studies, $30 \%$ serum was used for opsonization and neutrophils were pretreated with cytochalasin B.

Effect of heat inactivation on the opsonic activity in control and $C F$ sera. To examine the contribution of the complement system to the opsonic activity in serum, some samples were incubated at $56^{\circ} \mathrm{C}$ for $30 \mathrm{~min}$ to inactivate complement components. Studies using the four strains of mucoid $P$. aeruginosa indicated that they stimulated similar levels of superoxide anion production and showed similar patterns of activity when opsonized (data not shown). In subsequent experiments a single mucoid strain was chosen and a summary of the results obtained using control and CF sera to opsonize mucoid and nonmucoid $P$. aeruginosa is shown in Figure 2. In the absence of opsonins (Fig. 2 , right column), mucoid $P$. aeruginosa particles stimulated the release of significantly more superoxide anion $(2.16 \mathrm{nmol} \pm 1.32$, mean $\pm 1 \mathrm{SD})$ than the nonmucoid $(0.71 \mathrm{nmol} \pm 0.58$, mean \pm 1 SD) $P$. aeruginosa $(p<0.001)$. When control sera were used to opsonize nonmucoid $P$. aeruginosa particles (Fig. 2, top panel, left column), in all cases, heat inactivation lowered the opsonic activity. This decrease was about $50 \%$ and indicates that com-
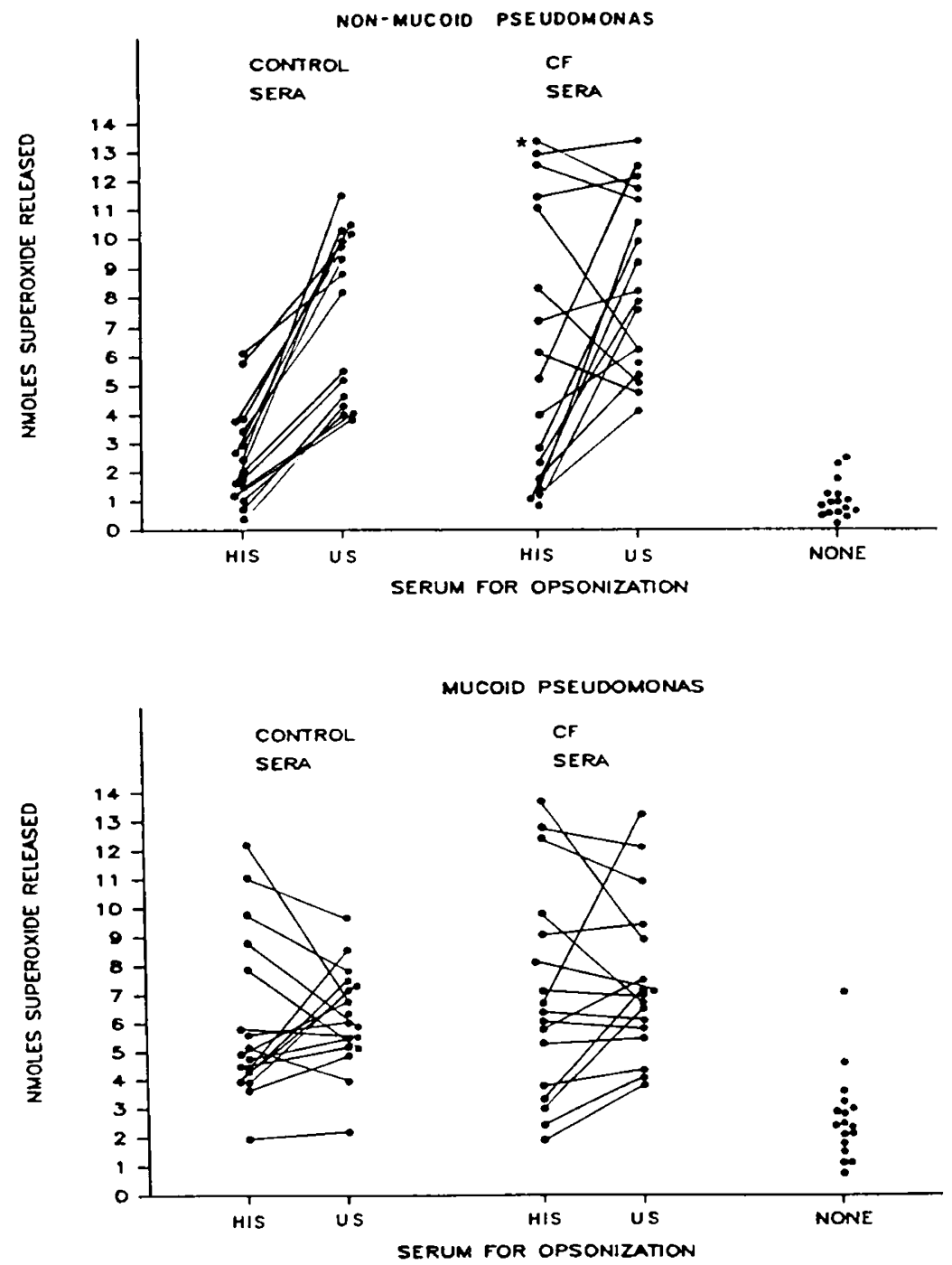

Fig. 2. Opsonic activity in serum from normals (CONTROL) and CF patients to nonmucoid and mucoid $P$. aeruginosa. $P$. aeruginosa particles were opsonized with either heat-inactivated serum (HIS), untreated serum (US), or PBS (NONE) and used to stimulate superoxide anion release from normal neutrophils which had been pretreated with $5 \mu \mathrm{g} / \mathrm{ml}$ cytochalasin B. Mean \pm SD superoxide anion released using nonmucoid $P$. aeruginosa opsonized with PBS (NONE), $0.71 \pm 0.58$; CONTROL-HIS, $2.62 \pm 1.75$; CONTROL-US, $7.36 \pm 2.87$; CF-HIS, 6.02 \pm 4.54 ; CF-US, $8.61 \pm 2.95$ or using mucoid $P$. aeruginosa opsonized with PBS (NONE), $2.16 \pm 1.32$; CONTROL-HIS, $6.05 \pm 2.86$; CONTROL-US, $6.12 \pm 1.8$; CF-HIS, $6.62 \pm 3.68$; CF-US, $7.2 \pm 2.71$. The asterisk indicates significant differences $(p<0.05)$ were observed between CF-HIS and CONTROLHIS using nonmucoid $P$. aeruginosa. 
plement-mediated activity accounts for about half of the opsonic activity of the serum. When heat-inactivated CF sera were used to opsonize the nonmucoid $P$. aeruginosa the responses observed were significantly higher than with heat inactivated control sera when compared with the paired $t$ test $(p=0.008)$. In addition, with five of the $16 \mathrm{CF}$ sera, more activity was observed in the heat-inactivated sample than in the original serum (Fig. 2, top panel, center column). Analysis of patient information on these five sera showed that all five patients were colonized with $P$. aeruginosa. In contrast, when mucoid $P$. aeruginosa was used, heat inactivation of the serum resulted in a higher response in seven of 17 control serum samples (Fig. 2, bottom panel, left column) Similarly, when CF sera were used to opsonize the mucoid $P$. aeruginosa, eight of the 16 samples showed higher activity in the heat-inactivated preparation (Fig. 2, bottom panel, center column). Of these eight sera, five were from patients colonized with $P$. aeruginosa. This pattern of higher in heatinactivated sera occurs predominantly in the patient population which is colonized with $P$. aeruginosa. This may therefore reflect a higher level of antibodies to $P$. aeruginosa which may become aggregated during heating and provide a better opsonic signal.
Comparison of serum from patients colonized with $P$. aeruginosa with those who were culture negative. In order to examine the effect of $P$. aeruginosa colonization on the opsonic activity measured by superoxide anion release, comparisons were made between patients who were colonized and those who were not. As shown in Figure 3, when the superoxide anion response using heat-inactivated serum from patients colonized with $P$. aeruginosa was compared to the serum from patients who were not colonized, significant differences were observed with both the mucoid and nonmucoid $P$. aeruginosa particles. With the mucoid $P$. aeruginosa a significant difference between the groups was observed when either heat-inactivated or untreated serum was used for opsonization. These data indicate that $\mathrm{CF}$ patients colonized with $P$. aeruginosa have greater opsonic activity in their serum which can cause the stimulation of superoxide anion release from neutrophils. This activity can be detected in heatinactivated serum when nonmucoid $P$. aeruginosa is used as the stimulus or in either heat-inactivated or normal serum when mucoid $P$. aeruginosa is used.

Correlation of opsonic activity with clinical status. Further comparisons were made between serum opsonic activity and the
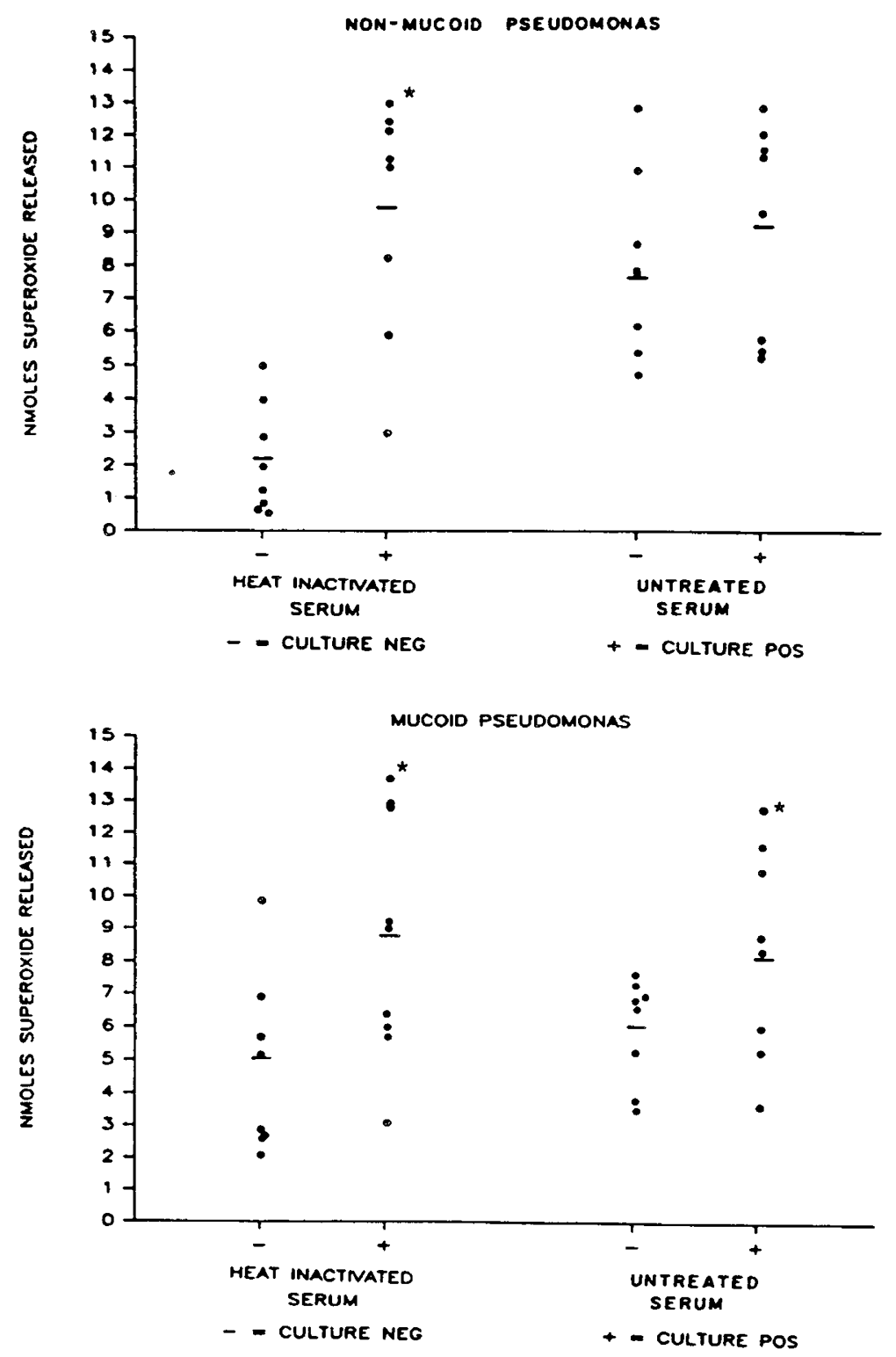

Fig. 3. Opsonic activity to nonmucoid or mucoid $P$. aeruginosa in heat-inactivated and normal serum from CF patients which were culture negative $(-)$ or culture positive $(+)$ for $P$. aeruginosa. Horizontal bars indicate sample means. Asterisks indicate significant differences $(p<0.05)$ were observed between the culture-positive and culture-negative groups. 
clinical status of the CF patients. No correlation with opsonic activity and clinical status was observed in patients who were not colonized with $P$. aeruginosa. However, with patients colonized with $P$. aeruginosa a negative correlation was observed between the opsonic activity measured by the production of superoxide anion and the Schwachman score (Fig. 4). The higher the Schwachman score the lower the opsonic activity. Similar correlations were observed using either the mucoid $(r=0.821)$ or nonmucoid ( $r=0.785) P$ aeruginosa. These data indicate that in patients colonized with $P$. aeruginosa the deterioration of the clinical status correlates with increased opsonic activity reflected in the oxidative stimulation of neutrophils.

\section{DISCUSSION}

$P$. aeruginosa is the major pathogen in CF. Colonization with mucoid strains of $P$. aeruginosa (17) as well as increased antibody titers to $P$. aeruginosa antigens have been associated with a poorer clinical state (6).

Why this is so is not entirely clear; so the present study examined opsonic factors in $C F$ serum with respect to their ability to stimulate the release of superoxide anion from neutrophils. The measurement of neutrophil oxygen radical production has been widely used as a method to quantify opsonic activity $(9,10)$. To examine this response specifically, cytochalasin B treatment of neutrophils was used to minimize differences in phagocytic uptake which have been observed between mucoid and nonmucoid strains of $P$. aeruginosa $(18)$ as well as to enhance the extracellular production of superoxide anion (15). Comparisons of mucoid and nonmucoid $P$. aeruginosa showed that without any opsonization, mucoid $P$. aeruginosa stimulated significantly more $\mathrm{O}_{2}^{-}$than nonmucoid. When control sera were used to opsonize nonmucoid $P$. aeruginosa, heat inactivation always lowered the level of $\mathrm{O}_{2}^{-}$generated. However, when mucoid $P$. aeruginosa was used, heat inactivation often increased the production of $\mathrm{O}_{2}^{-}$. Similar results were observed with $\mathrm{CF}$ sera with both mucoid and nonmucoid organisms. Further analysis indicated that these sera were primarily from patients who were colonized with $P$. aeruginosa. Because these patients are known to have high antibody titers to $P$. aeruginosa $(6)$, these data imply a role for specific antibody in this response and suggest that the increased activity seen in some heat-inactivated sera may be due to antibodies which have aggregated during heating and are more stimulatory. The greatest loss of activity after heat inactivation was observed in serum samples from culture negative patients using nonmucoid $P$. aeruginosa as the stimulus. These data suggest that heat labile complement components are more important in the absence of specific antibody when nonmucoid $P$. aeruginosa is involved. Heat inactivation of serum had less of an effect when mucoid $P$. aeruginosa were used. One explanation for these findings is that complement components (such as $\mathrm{C} 3 \mathrm{~b}$ ) may not play a role in the stimulation of $\mathrm{O}_{2}{ }^{-}$from neutrophils when mucoid forms of $P$. aeruginosa are used. This possibility is supported by studies by Wright and Silverstein (19) who have demonstrated that stimulation of $\mathrm{O}_{2}^{-}$ production in PMNs and monocytes results from interaction with $\mathrm{Fc}$ receptors rather than with $\mathrm{C} 3 \mathrm{~b}$ receptors. Our data indicate that mucoid $P$. aeruginosa stimulate neutrophils to a greater degree than nonmucoid $P$. aeruginosa in the absence of serum. Heat labile complement components may play a greater role in the opsonization of nonmucoid $P$. aeruginosa in the absence of antibodies since control sera and uninfected patient sera lost substantial activity upon heating. In contrast, heat inactivation had a lesser effect on opsonic activity with mucoid $P$. aeruginosa. This is consistent with reports that mucoid strains of $P$. aeruginosa are not as effective as nonmucoid strains in activating the complement cascade (18).

Comparisons of serum from patients colonized with $P$. aeruginos $a$ and those not colonized indicated a significantly higher opsonic activity in the colonized patients. This finding is in agreement with observations by LeBlanc et al. (20) who used a chemiluminescence assay to measure opsonic activity in CF sera to $P$. aeruginosa. This likely reflects higher antibody titers to $P$. aeruginosa in serum from colonized patients and indicates the functional ability of this antibody to stimulate oxygen radical production in neutrophils. This possibility is further substantiated by the observation of a correlation between the level of superoxide anion response and the clinical condition of the patient. Higher levels of superoxide anion release correlated with poor clinical conditions reflected by low Schwachman scores.

Together these findings support the model in which oxygen radical production is involved in the progression of the lung disease in CF. In this model, increased antibody titers resulting from chronic colonization with $P$. aeruginosa may result in enhanced oxygen radical production from neutrophils which migrate to the lung. These oxygen radicals may cause damage which prevents the eradication of the infection.

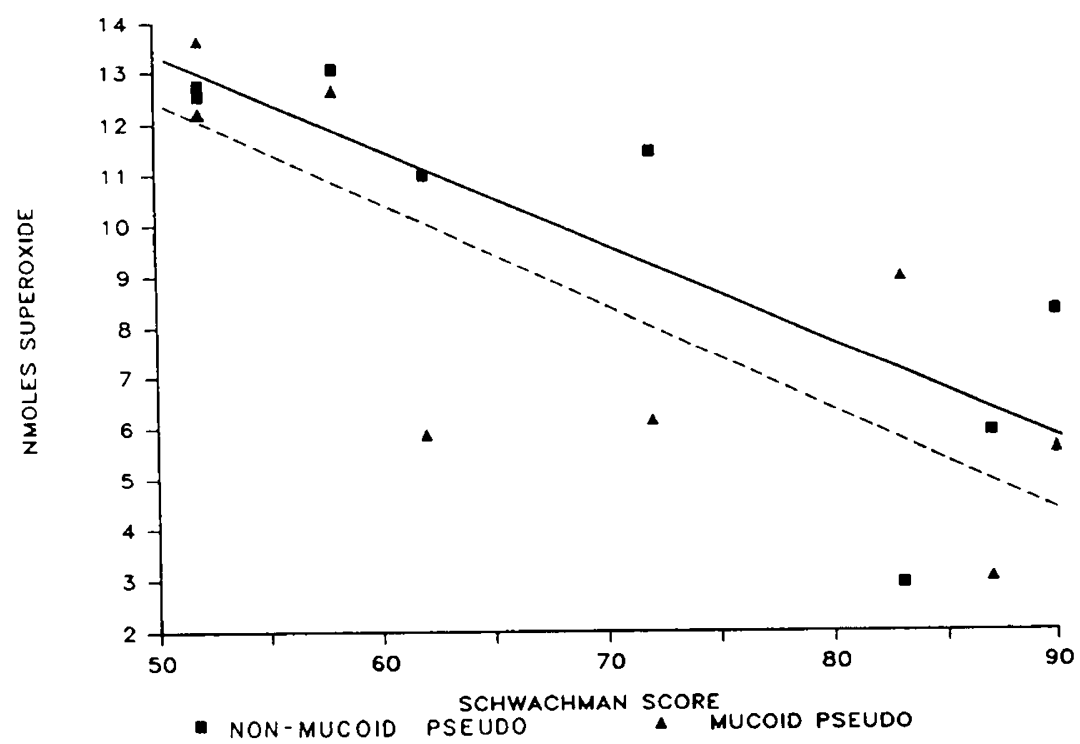

Fig. 4. Comparison of serum opsonic activity and clinical status measured by Schwachman scores of CF patients colonized with $P$. aeruginosa. Significant correlation $(p<0.05)$ were observed with nonmucoid $P$. aeruginosa $(r=-0.821(\square)$ and mucoid $P$. aeruginosa $(r=-0.785)(\mathbf{\Delta})$. 


\section{REFERENCES}

1. Pier GB 1985 Pulmonary disease associated with Pseudomonas aeruginosa in cystic fibrosis: current status of the host-bacterium interaction. J Infect Dis 151:575-580

2. Talamo RC, Schwartz RH 1984 Immunologic and allergic manifestations. In: Taussig LM (ed) Cystic Fibrosis. Thieme-Stratton, New York, pp 175-193

3. Moss RB 1983 Immunology of cystic fibrosis: immunity, immunodeficiency and hypersensitivity. In: Lloyd-Still JD (ed) Textbook of Cystic Fibrosis. John Wright, Boston, pp 109-151

4. Johnson FJ, Ward PA 1981 Role of oxygen metabolites in immune complex injury of lung. J Immunol 126:2365-2367

5. Matthews WJ. Williams M, Oliphant B, Geha R, Colten HR 1980 Hypogammaglobulinemia in patients with cystic fibrosis. N Engl J Med 302:245-249

6. Doring G, Hoiby N 1983 Longitudinal study of immune response to Pseudomonas aeruginosa antigens in cystic fibrosis. Infect Immun 42:197-201

7. Fick RB Jr, Naegel GP, Squier SU, Wood RE, Gee JBL, Reynolds HY 1984 Proteins of the cystic fibrosis respiratory tract. Fragmented immunoglobulin $\mathrm{G}$ opsonic antibody causing defective opsonophagocytosis. J Clin Invest $74: 236-248$

8. Rehm SE, Gross GN, Pierce AK 1980 Early bacterial clearance from murine lungs: species dependent phagocyte response. J Clin Invest 66:194-199

9. Allen RC, Lieberman MM 1984 Kinetic analysis of microbe opsonification based on stimulated polymorphonuclear leukocyte oxygenation activity. Infect Immun 45:475-482

10. Bellavite P, Dri P, Della Bianca V, Serra MC 1983 The measurement of superoxide anion production by granulocytes in whole blood. A clinical test for the evaluation of phagocyte function and serum opsonic capacity. Eur J
Clin Invest 13:363-368

11. Boyum A 1968 Isolation of mononuclear cells and granulocytes from human blood. Scand J Clin Lab Invest 21(suppl 97):77-88

12. Pick E, Mizel D 1981 Rapid microassays for the measurement of superoxide and hydrogen peroxide production by macrophages in culture using an automatic enzyme immunoassay reader. J Immun Methods 46:211-226

13. Massey V 1959 The microestimation of succinate and the extinction coefficient of cytochrome C. Biochem Biophys Acta 34:255-256

14. Schwachman H, Kulczycki LL 1958 Long-term study of one hundred five patients with cystic fibrosis. Am J Dis Child 96:6-15

15. Root RK, Metcalf JA 1977 Hydrogen peroxide release from human granulocytes during phagocytosis. Relationship to superoxide anion formation and cellular catabolism of hydrogen peroxide: studies with normal and cytochalasin B-treated ceils. J Clin Invest 60:1266-1279

16. Zigmond SH, Hirsch JG 1972 Effects of cytochalasin B on polymorphonuclear leucocyte locomotion, phagocytosis and glycolysis. Exp Cell Res 73:383-393

17. Kulczycki LL, Murphy TM, Bellanti JA 1978 Pseudomonas colonization in cystic fibrosis. A study of 160 patients. JAMA 240:30-31

18. Meshulam T, Obedeanu N, Merzbach D, Sobel JD 1984 Phagocytosis of mucoid and nonmucoid strains of Pseudomonas aeruginosa. Clin Immunol Immunopathol 32:151-165

19. Wright SD, Silverstein SC 1983 Receptors for C3b and C3bi promote phagocytosis but not the release of toxic oxygen from human phagocytes. $\mathbf{J}$ Exp Med 158:2016-2023

20. LeBlanc CMA, Bortolussi R, Issekutz AC, Gillespie T 1982 Opsonization of mucoid and non-mucoid Psuedomonas aeruginosa by serum from patients with cystic fibrosis assessed by a chemiluminescence assay. Clin Invest Med $5: 125-128$

\section{Announcement}

\section{THE ASSOCIATION OF MEDICAL SCHOOL PEDIATRIC DEPARTMENT CHAIRMEN, INC} proudly announces the

Pediatric Scientist Training Program 1987 Fellows

Name

Kendall Brown, M.D.

George T. Capone, M.D.

Darryl C. Longee, M.D.

Karen McAlmon, M.D.

Lasurie C. Miller, M.D.

Sharon A. Nachman, M.D.

Michael R. Narkewicz, M.D.

Scott A. Rivkees, M.D.
Sponsoring Department and Chairman

University of Texas, Dallas Joseph B. Warshaw, M.D.

Children's Hospital Medical Center, Cincinnati William K. Schubert, M.D.

Duke University

Samuel L. Katz, M.D.

Boston Children's Hospital David G. Nathan, M.D.

Tufts University

Jane G. Schaller, M.D.

New York Medical College

Edward Wasserman, M.D.

University of Colorado

Frederick C. Battaglia, M.D.

Harvard Medical School Donald M. Medearis, Jr., M.D.

and gratefully acknowledges the support and sponsorship of

National Institute of Child Health and Human Development

March of Dimes Birth Defects Foundation

Cystic Fibrosis Foundation

St. Jude Children's Research Hospital 